$>$ La fonction des cellules dendritiques (CD) est d'amorcer et d'orienter la réponse adaptative grâce à leur capacité à reconnaître, à l'aide des récepteurs de type PRR (pattern recognition receptors), un nombre restreint de structures moléculaires propres aux micro-organismes et communes à de nombreux agents pathogènes. La stratégie de ces sentinelles n'est pas de reconnaître toute la gamme des millions de motifs antigéniques existants mais un petit nombre d'entre eux et d'intégrer cette information afin d'aider le système adaptatif à faire la distinction entre peptides du soi et peptides dangereux. Nous verrons dans cette revue que le virus de l'hépatite $C$ interrompt la signalisation en aval de certains PRR, ce qui lui permet d'échapper à la détection par l'immunité innée et d'inhiber la capacité des CD à stimuler une réponse adaptative antivirale. <

\section{Comment le virus de l'hépatite C détourne la réponse immunitaire adaptative orchestrée par les cellules dendritiques}

Ian Gaël Rodrigue-Gervais, Daniel Lamarre

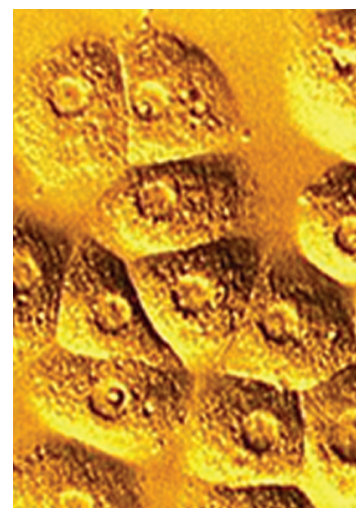

I.G. Rodrigue-Gervais :

Laboratoire

d'immunovirologie, CR-CHUM, Hôpital Saint-Luc, Montréal (Québec), H2X IPI Canada. Département de microbiologie et immunologie,

Université de Montréal, CP 6128 , Succursale centre-ville, Montréal (Québec) H3C 3J7 Canada.

D. Lamarre: Directeur

du Laboratoire

d'immunovirologie, CR-CHUM,

Hôpital Saint-Luc, Montréal

(Québec), H2X IP1 Canada.

un éventail bien plus large de PRR (pathogen recognition receptors) pour alerter l'hôte de la présence d'agents pathogènes (voir Tableau 1). Kato et al. [3] montrent que les CDM produisent égale-

Département de médecine et Institut de recherche en immunologie et en cancérologie, faculté de médecine, Université de Montréal, CP 6128, Succursale centre-ville, Montréal (Québec) H3C 3J7 Canada. daniel.lamarre@umontreal.ca ment des IFN- $\alpha$ en réponse à la détection d'une infection virale et qu'elles le font en activant le facteur 3 de régulation de l'IFN (IRF3) via RIG-I (retinoic acid-inducible gene-I). En revanche, les CDP sont dépourvues de RIG-I et seul le système TLR proprement dit est utilisé. II existe de nombreuses façons d'activer les CD (ce qu'illustre le Tableau II), par exemple via les cytokines pro-inflammatoires [interleukine (IL)- $1 \beta$, TNF (tumor necrosis factor)- $\alpha$, etc.], le lipopolysaccharide (LPS), I'ARN40 simple brin du virus de l'immunodéficience humaine $(V I H)$. Les partenaires moléculaires et la cascade signalétique (PRR et adaptateurs, voir Tableau II) étant différents dans chaque situation [4], chacune 


\begin{tabular}{|c|c|c|c|c|}
\hline \multirow{12}{*}{ 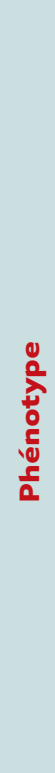 } & & CDM & CDP* & Monocyte \\
\hline & CD3 & - & - & - \\
\hline & CD4 & + & ++ & + \\
\hline & CD8 & - & - & - \\
\hline & CDIlc & + & 10 & + \\
\hline & CD14 & 10 & - & + \\
\hline & CD16 & - & - & $+/-$ \\
\hline & CD19 & - & - & - \\
\hline & CD33 & + & 10 & + \\
\hline & CD123/IL3R $\alpha$ & + & ++ & $+/-$ \\
\hline & CD303 & - & + & - \\
\hline & HLA-DR & ++ & + & + \\
\hline \multirow{5}{*}{ 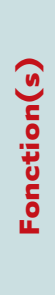 } & IFN- $\alpha / \beta$ & + & +++ & + \\
\hline & Cytokines & ++ & + & + \\
\hline & Phagocytose & + & - & ++ \\
\hline & Mémorisation & + & - & - \\
\hline & Polarisation ${ }^{\star}$ & + & ++ & - \\
\hline
\end{tabular}

de ces voies d'activation doit donc être analysée de manière spécifique. À titre indicatif, les CDM activées par le TNF- $\alpha$ sont propres à stimuler l'expansion clonale de lymphocytes T mémoires CD4 spécifiques du virus influenza chez des patients infectés avec le virus de l'hépatite C (VHC) [5]. Toutefois, ce résultat ne renseigne pas sur le potentiel d'activation des CD en réponse aux motifs moléculaires associés aux pathogènes (PAMP) reconnus par les PRR spécialisés dans la détection de constituants viraux (ex. : ARN double-brin par le TLR3). Ces derniers sont paradoxalement restés inexplorés par l'étude de Longman et al. [5]. Malgré une exécution prudente des expériences, la conclusion que ces auteurs tirent de leurs observations se résume à une capacité fonctionnelle normale des CDM circulantes chez des patients atteints d'une hépatite $C$ chronique [5]. Rappelons que, si d'autres cellules comme les lymphocytes B, les CDP ou les monocytes/macrophages expriment des PRR et peuvent également présenter des antigènes à des cellules T activées, les CDM, elles, sont les seules cellules présentatrices d'antigènes capables, in vivo, d'activer pleinement l'induction de lymphocytes T CD8. D'ailleurs, la déplétion in vivo des CDM empêche l'induction d'une réponse CTL (réponse T cytotoxique) chez des souris soumises à une infection virale $[6,7]$.
Tableau I. Phénotype et caractéristiques fonctionnelles des cellules présentatrices d'antigène isolées du sang périphérique. *Les CDP coopèrent avec les CDM in vivo en induisant la différenciation des lymphocytes $T$ non polarisés mais préalablement activés par les CDM ; lo : expression faible.

Notre exposé poursuit trois objectifs. En premier lieu, il illustre comment les CD sont au cœur des relations qui façonnent l'immunité adaptative. Ensuite, il met en évidence les nombreuses données expérimentales en faveur d'une interaction virus-hôte négative entre les CD et le VHC. Enfin, il apporte des arguments en faveur de l'hypothèse de l'existence d'un défaut sélectif des CD ciblées par le VHC.

\section{Les cellules dendritiques sont-elles des cibles du VHC?}

Les données in vivo en faveur de la thèse selon laquelle le VHC esquiverait la réponse immunitaire de l'hôte infecté grâce à sa capacité d'inhiber la fonction des CDM sont plutôt rares. Le rôle physiologique de ces cellules dans l'infection VHC a surtout été inféré à partir d'études de cohortes hétérogènes (c'est-à-dire composées de patients présentant divers génotypes et différents antécédents thérapeutiques) et avec des systèmes de dérivation in vitro des monocytes en CD (Mo-CD). Leurs résultats sont contradictoires [8] : certaines études ont rapporté des défauts reliés à une infection des Mo-CD, d'autres n'ont tout simplement pas observé ce phénomène. D'ailleurs, aucun ARN VHC n'a été détecté dans des Mo-CD préparés à partir de monocytes de chimpanzés chroniquement infectés, et ce même en utilisant un essai avec une sensibilité de 2500 copies d'ARN viral par $10^{6}$ Mo-CD [9]. L'idée que les CD ne sont pas infectées, que leurs fonctions de présentation d'antigènes ne sont pas altérées et que les défauts observés sont une conséquence passive de pathologies secondaires émanant d'une virémie plasmatique élevée s'est rapidement imposée. Toutefois, les différences de réponse à l'infection VHC du chimpanzé, modèle animal permissif, et de l'homme limitent la comparaison. De plus, une semaine de culture avec des doses élevées de GM-CSF (granulocyte macrophage colony stimulating factor) et d'IL-4 pourrait avoir amélioré les capacités présentatrices d'antigènes de précurseurs non défectifs dans le système in vitro et permis de surmonter le défaut immunologique présent in vivo dans une sous-population de CD [10]. Il est à noter que la présence d'ARN viraux du VHC a été détectée, tout comme celle de sa matrice de réplication, dans les CD primaires directement isolées (sans manipulation in vitro) chez des patients porteurs d'une infection chronique par le VHC [1012]. La proportion de CD sanguines infectées est faible, $0,1 \%$ à $1 \%$, de même que les niveaux d'ARN du VHC mesurés dans ces CD, estimée à moins d'une copie par cellule. Cependant, même ces faibles nombres de CD infectées sont susceptibles d'affecter la stimulation antigènespécifique lors d'une infection par le VIH [13].

Si un certain consensus existe quant à la présence d'ARN viral dans les $C D$ de sujets virémiques, la conséquence immunologique de la détection de ces séquences demeure ambiguë. De fait, les deux phénomènes, circulation de CD infectées et chronicité, sont en effet corrélés mais non strictement dépendants. Dans certains cas, par exemple 


\begin{tabular}{|c|c|c|c|c|c|c|c|}
\hline \multirow{12}{*}{$\frac{\alpha}{\alpha}$} & & CDM & CDP & Monocyte & Adaptateur & Localisation & Ligand PAMP \\
\hline & $R I G-I^{(1)}$ & + & - & + & IPS-1 & C & $\begin{array}{l}\text { ARN double brin, } \\
\text { ARN simble brin 5'ppp }\end{array}$ \\
\hline & TLRl/6 & + & 10 & + & MyD88 & $S$ & Lipoprotéines triacylées \\
\hline & TLR2 & + & - & ++ & MyD88 & $S$ & $\begin{array}{c}\text { Peptidoglycane, acide lipotéichoïque, } \\
\text { heatshock protein } 90\end{array}$ \\
\hline & $\operatorname{TLR} 3^{(1)(2)}$ & ++ & - & + & TRIF & $\varepsilon$ & ARN double brin \\
\hline & $\mathrm{TLR} 4^{(1)(2)}$ & + & - & ++ & $\begin{array}{l}\text { TRIF } \\
\text { MyD88 }\end{array}$ & $S / \varepsilon$ & LPS, enveloppes virales \\
\hline & TLR5 & + & - & + & MyD88 & $S$ & Flagelline \\
\hline & $\operatorname{TLR}^{(1)}$ & - & ++ & - & MyD88 & $\varepsilon$ & $\mathrm{R}-837,3 \mathrm{M}-011$ \\
\hline & $\operatorname{TLR}^{(1)(2)}$ & ++ & - & + & MyD88 & $\varepsilon$ & ARN40 simple brin, 3M-002 \\
\hline & TLR9(1)(2) & - & ++ & - & MyD88 & $\varepsilon$ & ADN CpG non méthylé \\
\hline & TLR10 & + & 10 & + & MyD88 & $?$ & $?$ \\
\hline & TLRIl & + & - & + & MyD88 & $S$ & Profiline \\
\hline
\end{tabular}

Tableau II. Partenaires moléculaires et cascade de signalisation des cellules présentatrices d'antigène isolées du sang périphérique. ${ }^{\star} \mathrm{C}$ : cytosol ; $S$ : surface cellulaire; $\varepsilon$ : endosome. Les mots écrits en rouge correspondent aux TLR qui utilisent MyD88 comme adaptateur signalétique. ${ }^{(1)}$ PRR impliqué dans la détection d'infections virales. ${ }^{(2)}$ Mode d'action ne nécessitant pas une réplication virale active.

chez les chimpanzés et, dans une moindre mesure, chez l'être humain, la circulation de CD infectées et dysfonctionnelles peut être indétectable alors que la virémie plasmatique demeure paradoxalement très élevée $[10,14]$. Toutefois, dans la majorité des cas chez l'être humain, la présence du génome du VHC dans les CD, mesurée avec les techniques sensibles de RT-PCR, n'est détectable que chez les patients chroniquement infectés [10] et sa diminution sous traitement IFN- $\alpha$ est associée à une restauration des fonctions allostimulatrices de ces cellules [15]. De là l'établissement d'un paradigme intégrant le sort et la fonction des CD au cours de l'infection au VHC en deux états mutuellement exclusifs (dysfonction en opposition à normalité).

\section{Réservoir VHC dans les CD : un flirt bénéfique pour le virus?}

Nous avons démontré que les niveaux d'expression d'IL-12 et de TNF- $\alpha$ suivant l'engagement du TLR3 et du TLR4 dans les CDM sont négativement corrélés avec les niveaux d'ARN génomique du VHC présent dans les $C D$ circulantes de patients virémiques [10]. L'atténuation de la réponse innée n'est corrélée ni avec des déficits dans la fréquence des CDM chez ces patients, ni avec la virémie ou les niveaux d'alanine transaminase et d'aspartate aminotransférase. Combinées, nos données indiquent que les interactions virus-hôte entre le VHC et les CDM et la perte de fonction des TLR utilisant l'adaptateur TRIF (Toll/IL-1-domain-containing adapter-inducing IFN- $\beta$ ) ne sont pas des phénomènes indépendants. D'autres recherches ont confirmé la réduction importante de la capacité des CDM à synthétiser l'IL-12, le TNF- $\alpha$ et I'IFN- $\beta$ dans le contexte de l'infection par le VHC après engagement du TLR3 et plus récemment du TLR4 et de l'hélicase cytoplasmique RIG-I. Tous ces récepteurs déclenchent une réponse d'immunité innée en fixant l'ARN viral (les motifs double brin et 5'-triphosphate) ou les glycoprotéines de l'enveloppe virale (voir Tableau / et II) [16-18]. À I'appui de ces observations chez les patients virémiques, les CD issues de souris rrif $^{-/-}$[19] ou porteuses d'une protéine TRIF mutée [20], et des souris Ips-1 (IFN- $\beta$ promoter

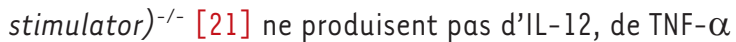
ou d'IFN- $\beta$ en réponse à une activation des TLR3/TLR4 et de RIG-I respectivement. Ainsi, les voies contrôlées par TRIF et IPS-l paraissent indispensables à l'induction de ces cytokines dans ces cellules lorsqu'elles détectent I'ARN viral. D'ailleurs, la restauration de la fonctionnalité du TLR4 in vitro par des inhibiteurs ciblant la NS3/4A protéase (BILN2061) et la NS5B ARN polymérase du VHC nous a permis de montrer que l'activité protéolytique de la NS3 et la réplication virale interfèrent avec l'activation de la voie TRIF-dépendante dans ces CDM infectées [22]. La NS3, son cofacteur NS4A et la NS5B sont des protéines virales non structurales codées par le VHC qui assurent les fonctions enzymatiques essentielles à la réplication du génome viral. Le ciblage, par l'activité protéase de la NS3/4A, de l'adaptateur TRIF au niveau de sa cystéine 372 et d'IPS-1 sur sa cystéine 508 [23] 


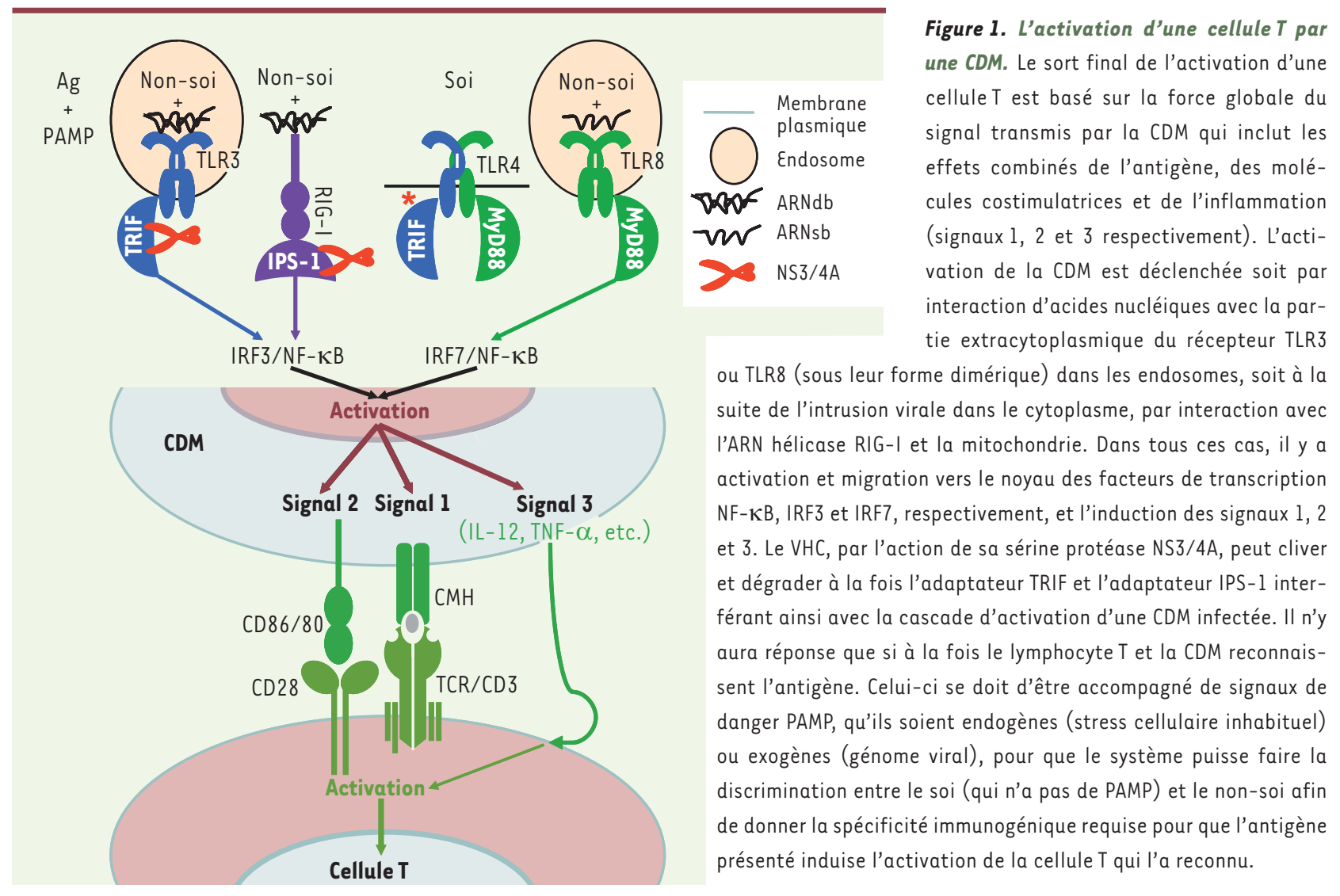

a été décrit récemment comme moyen d'inhibition de la voie en aval du TLR3 et de RIG-I. Cette activité protéolytique est très sensible à l'inhibiteur BILN206l qui restaure complètement, après seulement 24 heures de traitement, les fonctions antivirales dans des hépatocytes infectés in vitro avec le VHC [24]. II semblerait alors que l'un des rôles physiologiques de la protéase NS3/4A dans des cellules infectées soit de prévenir l'induction d'une réponse inflammatoire déclenchée par la voie du TLR3 (et du TLR4) dépendante de TRIF et celle de RIG-1 dépendante d'IPS-1 pour permettre au VHC d'échapper à la détection par l'immunité innée (Figure 1).

\section{L’effet sur la réponse adaptative anti-VHC}

Cette stratégie d'évasion mise en œuvre par le VHC pour éviter la détection de ses motifs moléculaires associés aux pathogènes (PAMP) par les CD est susceptible d'exercer une pression inhibitrice sur l'apprêtement et la présentation appropriée des antigènes du VHC aux lymphocytes T spécifiques (voir Figure 1). Effectivement, chez la souris, l'invalidation des adaptateurs spécifiques à ces PRR - Myd88 (myeloid differentiation gene) [25], Traf6 (TNF receptor-associated factor) [26], Tram (TLR-associated molecule) [27], Trif [19] et Ips-1
[21] - conduit à des anomalies de fonctionnement des CD en lien avec l'inactivation du système adaptatif [28]. Les antigènes qui sollicitent le système immunitaire dans des conditions d'absence de signaux de danger conduisent à une anergie, un épuisement fonctionnel et/ou une élimination clonale (au moins des clones les plus affins) de la réponse cellulaire T. À terme, ceci aboutit, dans tous les cas, à un état de tolérance vis-à-vis de l'antigène reconnu [29]. Cette tolérance est strictement spécifique de l'antigène et récessive, de sorte que seuls les lymphocytes $T$ ayant effectivement reconnu des peptides présentés par des CD inactives sont inactivés [30]. Or, pour expliquer la spécificité du défaut de l'immunité anti-VHC, certains auteurs ont cru devoir écarter les $C D$, bien que des signaux provenant de ces dernières puissent être essentiels pour l'activation des cellules T. L'argument invoqué était qu'un tel mécanisme de persistance virale exigerait l'infection d'un pourcentage significatif de CD in vivo (loin des $0,1 \%$ à $1 \%$ rapportés pour les $C D$ circulantes) et que cela mènerait invariablement à une immunosuppression généralisée [31]. Des études récentes estiment que cette argumentation est en partie sans fondement, notamment parce que :

- la fréquence globale des CDM dysfonctionnelles est très faible (estimée à moins de $3 \%$ des CD sanguines [10]), indiquant l'absence de défauts globaux dans la reconnaissance innée des signaux de danger; - il n'est pas question d'une diminution généralisée de la réponse des PRR dans tous les types cellulaires et toutes les voies de signalisation connues (du moins celles étudiées), mais plutôt d'une inhibition 
spécifique de la détection des ligands empruntant les voies relayées par TRIF et IPS-1 dans les CDM infectées [18] puisque la production de cytokines en réponse à l'activation du TLR8 (voie dépendante de MyD88) demeure intacte [22] et que les monocytes de ces patients restent fonctionnels [10].

Récemment, l'activation de CDM par la reconnaissance directe des PAMP (qui habituellement sont associés de façon covalente à l'antigène duquel ils dérivent), plutôt que l'activation indirecte par des cytokines inflammatoires (ex.: TNF- $\alpha$ ), s'est avérée être un prérequis incontournable in vivo pour qu'il y ait induction d'effecteurs mémoire T fonctionnels $[28,32]$. On sait maintenant que l'activation indirecte des CDM qui n'ont pas encore rencontré l'agent pathogène (donc ni PAMP, ni antigènes) a pour conséquence de limiter leur participation à la présentation d'antigènes, principalement en arrêtant la capture des antigènes exogènes par phagocytose et en empêchant la formation de nouveaux complexes $\mathrm{CMH}-\mathrm{II}$-peptide $[33,34]$. En extrapolant à partir des travaux préalablement cités, on peut considérer que le nombre de CDM qui répondent à un pathogène donné est probablement petit si I'on considère que toutes les CDM qui présenteront aux lymphocytes $T$ des antigènes d'une spécificité donnée devront d'abord avoir été en contact direct avec les composants dudit pathogène. Or, dans le scénario qui nous intéresse dans cette synthèse, cela ouvre la voie à une attaque par la protéase NS3/4A qui ne peut alors interférer qu'avec les fonctions des CDM ciblées, celles-là même qui présenteront les antigènes du VHC aux lymphocytes. Par conséquent, cela laisse certainement assez de $C D$ en périphérie prêtes à répondre à de nouvelles agressions par d'autres agents infectieux. Ainsi le VHC peut-il échapper à la réponse immune dirigée contre ses antigènes, sans toutefois induire chez l’hôte infecté une immunosuppression généralisée.

\section{Conclusion}

Si le schéma de l'existence de CD fonctionnellement normales dans l'infection au VHC reste encore valide pour beaucoup, les résultats des recherches récentes exposés ici suggèrent qu'il est voué à disparaître. $\varepsilon n$ effet, les données accumulées dans les publications scientifiques au cours des trois dernières années fournissent désormais une base empirique à un modèle actuellement peu privilégié dans lequel l'affaiblissement de l'immunité innée à l'échelle des CD lors d'une infection virale participerait à l'échec de l'immunité adaptative spécifique du VHC. Dans ce modèle, la qualité des réponses antivirales dépend de la capacité qu'ont les CD à intégrer l'information issue du système inné et à la présenter aux lymphocytes T. Ainsi, il apparaît pertinent aujourd'hui de reconsidérer les CD comme des intermédiaires-clés entre le VHC et la qualité des réponses adaptatives développées contre ce virus. $\diamond$

\section{SUMMARY}

Hepatitis C virus subverts pattern recognition receptors-mediated control of adaptative immunity orchestrated by dendritic cells Chronic hepatitis C virus (HCV) is a liver-borne infectious disease that remains a major global health threat. The mechanisms whereby HCV evades the host's immune defences and establishes persistent infec- tion remain elusive; but they likely require a complex and coordinated interruption of the interplay between innate and adaptive immune actors. This review discusses the concept that HCV evades the host's immune response to its components partly because of its ability to inactivate the major orchestrator of the adaptive immune response - the DCs. It argues that DCs constitute an immunologically relevant cellular viral host actively targeted by HCV. This targeting disrupts TRIF- and IPS-1-dependent but not MyD88-coupled pathogen recognition receptors (PRR) sensing pathways in these infected cells to foil the networks by which innate immunity to HCV is translated into virus-specific adaptive immune-mediated host resistance. Thus, as a culprit, this cell-specific and numerically restrained DC defect offers a promising field of investigation in which to study and understand the $\mathrm{HCV}$-restricted nature of the deficit in cellular immunity in persistently infected individuals who have otherwise normal immune functions to unrelated pathogens. In this model, protective immunity is contingent on proper processing and delivery of danger signals by DCs presenting HCV antigens. $\diamond$

\section{GLOSSAIRE}

CD : cellule dendritique

CDM : cellule dendritique myéloïde

CDP : cellule dendritique plasmacytoïde

CMH-II: complexe majeur d'histocompatibilité de classe II

GM-CSF : granulocyte macrophage colony stimulating factor

IFN : interféron

IL : interleukine

IPS- 1 : IFN- $\beta$ promoter stimulator 1

IRF3 : facteur 3 de régulation de l'interféron

LPS : lipopolysaccharide

Mo-CD: cellule dendritique dérivée d'un précurseur monocytaire

MyD88 : myeloid differentiation gene 88

PAMP : pathogen associated molecular patterns

RIG-I : retinoic acid-inducible gene-I

TLR : Toll-like receptor

TNF : tumor necrosis factor

TRAF6 : TNF receptor-associated factor 6

TRAM : TLR-associated molecule

TRIF : Toll/IL-1-domain-containing adapter-inducing

IFN- $\beta$

VHC : virus de l'hépatite $C$

VIH : virus de l'immunodéficience humaine

\section{CONFLIT D'INTÉRÊTS}

Les auteurs déclarent n'avoir aucun conflit d'intérêts concernant les données publiées dans cet article. 


\section{REMERCIEMENTS}

Nous remercions tous les membres de notre laboratoire pour les discussions et les idées qui ont nourri cet article. Les recherches sous-jacentes à cet article ont bénéficié d'un financement de la part de l'Inserm et des instituts de recherche en santé du Canada (IRSC).

\section{RÉFÉRENCES}

1. Dzionek A, Sohma Y, Nagafune J, et al. BDCA-2, a novel plasmacytoid dendritic cell-specific type II C-type lectin, mediates antigen capture and is a potent inhibitor of interferon alpha/beta induction. J Exp Med 2001 ; $194:$ : 1823-34.

2. Liu YJ. IPC: professional type 1 interferon-producing cells and plasmacytoid dendritic cell precursors. Annu Rev Immunol 2005 ; 23 : 275-306.

3. Kato $\mathrm{H}$, Sato $\mathrm{S}$, Yoneyama $\mathrm{M}$, et al. Cell type-specific involvement of RIG-I in antiviral response. Immunity $2005 ; 23: 19-28$.

4. Palm NW, Medzhitov R. Pattern recognition receptors and control of adaptive immunity. Immunol Rev 2009; 227 : 221-33.

5. Longman RS, Talal AH, Jacobson IM, et al. Normal functional capacity in circulating myeloid and plasmacytoid dendritic cells in patients with chronic hepatitis C. J Infect Dis $2005 ; 192$ : 497-503.

6. Sapoznikov A, Fischer JA, Zaft T, et al. Organ-dependent in vivo priming of naive $\mathrm{CD}^{+}$, but not $\mathrm{CD} 8^{+}$, T cells by plasmacytoid dendritic cells. J Exp Med $2007 ; 204: 1923-33$.

7. Jung $S$, Unutmaz D, Wong $P$, et al. In vivo depletion of $C D 1 l c^{+}$dendritic cells abrogates priming of $C D 8^{+} T$ cells by exogenous cell-associated antigens. Immunity 2002; 17 : 211-20.

8. Longman RS, Talal AH, Jacobson IM, et al. Presence of functional dendritic cells in patients chronically infected with hepatitis C virus. Blood 2004 103 : 1026-9.

9. Larsson M, Babcock $\varepsilon$, Grakoui A, et al. Lack of phenotypic and functional impairment in dendritic cells from chimpanzees chronically infected with hepatitis C virus. J Virol $2004 ; 78$ : 6151-61.

10. Rodrigue-Gervais IG, Jouan L, Beaulé G, et al. Poly (I:C) and LPS innate sensing functions of circulating human myeloid dendritic cells are affected in vivo in hepatitis C virus-infected patients. J Virol 2007; $81: 5537-46$.

11. Goutagny N, Fatmi A, De Ledinghen V, et al. Evidence of viral replication in circulating dendritic cells during hepatitis $C$ virus infection. J Infect Dis $2003 ; 187$ : 1951-8.

12. Tsubouchi $\varepsilon$, Akbar SM, Horiike $N$, et al. Infection and dysfunction of circulating blood dendritic cells and their subsets in chronic hepatitis $C$ virus infection. J Gastroenterol 2004 ; 39 : 754-62.

13. Granelli-Piperno A, Shimeliovich I, Pack M, et al. HIV-1 selectively infects a subset of nonmaturing BDCAl-positive dendritic cells in human blood. J Immunol 2006; 176 : 991-8.

14. Rollier C, Drexhage JA, Verstrepen BE, et al. Chronic hepatitis C virus infection established and maintained in chimpanzees independent of dendritic cell impairment. Hepatology $2003 ; 38$ : 851-8.
15. Tsubouchi $\varepsilon$, Akbar SM, Murakami H, et al. Isolation and functional analysis of circulating dendritic cells from hepatitis C virus (HCV) RNA-positive and HCV RNA-negative patients with chronic hepatitis C: role of antiviral therapy. Clin Exp Immunol 2004 ; 137 : 417-23.

16. Averill L, Lee WM, Karandikar NJ. Differential dysfunction in dendritic cell subsets during chronic HCV infection. Clin Immunol $2007 ; 123: 40-9$.

17. Della Bella S, Crosignani A, Riva A, et al. Decrease and dysfunction of dendritic cells correlate with impaired hepatitis $\mathrm{C}$ virus-specific $\mathrm{CD} 4^{+} \mathrm{T}$-cell proliferation in patients with hepatitis $\mathrm{C}$ virus infection. Immunology 2007 ; $121: 283-92$.

18. Miyazaki M, Kanto T, Inoue M, et al. Impaired cytokine response in myeloid dendritic cells in chronic hepatitis $C$ virus infection regardless of enhanced expression of Toll-like receptors and retinoic acid inducible gene-I. J Med Virol 2008; $80: 980-8$.

19. Yamamoto M, Sato S, Hemmi H, et al. Role of adaptor TRIF in the MyD88-independent toll-like receptor signaling pathway. Science 2003; 301:640-3.

20. Hoebe K, Du X, Georgel P, et al. Identification of Lps2 as a key transducer of MyD88-independent TIR signalling. Nature $2003 ; 424$ : 743-8.

21. Kumar H, Koyama S, Ishii KJ, et al. Cooperation of IPS-1- and TRIF-dependent pathways in poly IC-enhanced antibody production and cytotoxic T cell responses. J Immunol 2008 ; $180: 683-7$.

22. Rodrigue-Gervais IG, Rigsby H, Jouan L, et al. Dendritic cell inhibition is connected to exhaustion of $C D 8^{+} T$ cell polyfunctionality during chronic hepatitis C virus infection. J Immunol 2010 ; $184: 3134-44$.

23. Li K, Foy $\varepsilon$, Ferreon JC, et al. Immune evasion by hepatitis C virus NS3/4A protease-mediated cleavage of the Toll-like receptor 3 adaptor protein TRIF. Proc Natl Acad Sci USA 2005; $102: 2992-7$.

24. Johnson CL, Owen DM, Gale M. Functional and therapeutic analysis of hepatitis C virus NS3.4A protease control of antiviral immune defense. J Biol Chem 2007; 282 : 10792-803.

25. Pasare C, Medzhitov R. Toll-dependent control mechanisms of CD4 T cell activation. Immunity $2004 ; 21: 733-41$.

26. Kobayashi T, Walsh PT, Walsh MC, et al. TRAF6 is a critical factor for dendritic cell maturation and development. Immunity $2003 ; 19: 353-63$.

27. Yamamoto $M$, Sato $S$, Hemmi $H$, et al. TRAM is specifically involved in the Toll-like receptor 4-mediated MyD88-independent signaling pathway. Nat Immunol $2003 ; 4$ : 1144-50.

28. Spörri R, Reis e Sousa C. Inflammatory mediators are insufficient for full dendritic cell activation and promote expansion of CD4+ T cell populations lacking helper function. Nat Immunol 2005; $6: 163-70$.

29. Steinman RM, Hawiger D, Nussenzweig MC. Tolerogenic dendritic cells. Annu Rev Immunol 2003; $21: 685-711$.

30. Probst HC, McCoy K, Okazaki T, et al. Resting dendritic cells induce peripheral CD8 ${ }^{+} \mathrm{T}$ cell tolerance through PD-1 and CTLA-4. Nat Immunol $2005 ; 6: 280-6$.

31. Dustin LB, Rice CM. Flying under the radar: the immunobiology of hepatitis C. Annu Rev Immunol $2007 ; 25$ : 71-99.

32. Nolte MA, Leibundgut-Landmann $S$, Joffre 0 , et al. Dendritic cell quiescence during systemic inflammation driven by LPS stimulation of radioresistant cells in vivo.J Exp Med 2007 ; $204: 1487-501$.

33. Wilson NS, Behrens GM, Lundie RJ, et al. Systemic activation of dendritic cells by Toll-like receptor ligands or malaria infection impairs cross-presentation and antiviral immunity. Nat Immunol $2006 ; 7: 165-72$.

34. Young LJ, Wilson NS, Schnorrer P, et al. Dendritic cell preactivation impairs MHC class II presentation of vaccines and endogenous viral antigens. Proc Natl Acad Sci USA 2007 ; $104: 17753-8$.

\section{TIRÉS À PART}

D. Lamarre

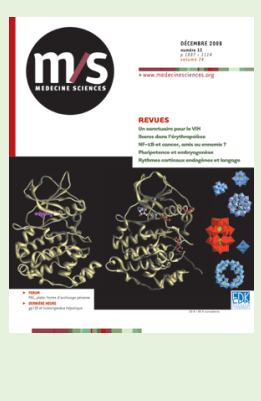

